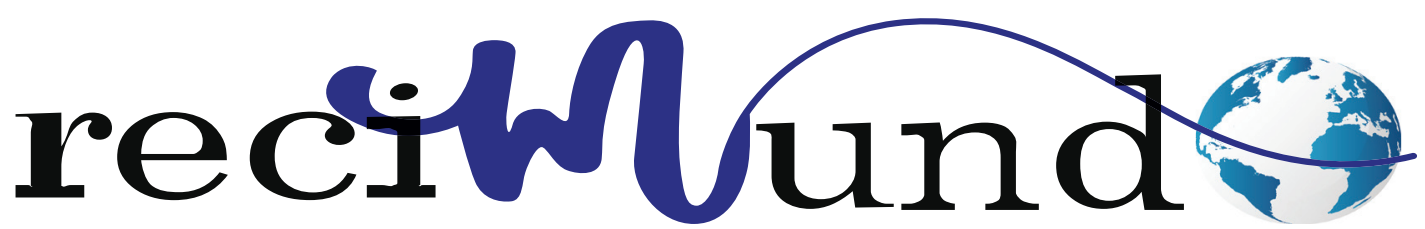

Revista Científica Mundo de la Investigación y el Conocimiento

DOI: 10.26820/recimundo/5.(1).enero.2021.153-163

URL: http://recimundo.com/index.php/es/article/view/994

EDITORIAL: Saberes del Conocimiento

REVISTA: RECIMUNDO

ISSN: 2588-073X

TIPO DE INVESTIGACIÓN: Artículo de Investigación CÓdigo UNESCO: 32 Ciencias Médicas

PAGINAS: $153-163$

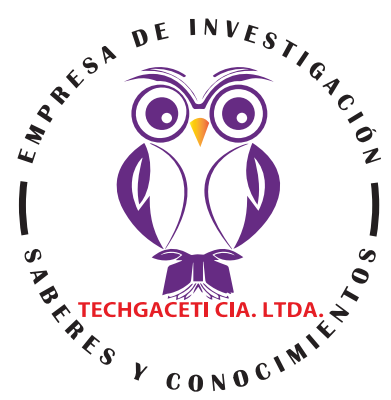

\title{
Evaluación de predictores de vía aérea difícil en el paciente pediátrico
}

Evaluation of predictors of difficult airway in the pediatric patient Avaliação de preditores de vias aéreas difíceis no paciente pediátrico Flor María Pancha Ramos'; Inés Tatiana Cevallos Pacheco²; Rosa Dalila López Samanieg03; Dipaola Pamela Pino Vaca ${ }^{4}$

RECIBIDO: 28/11/2020 ACEPTADO: 06/12/2020 PUBLICADO: 31/01/2021

1. Médico Especialista en Anestesiología; Investigador Independiente; Quito, Ecuador; dr.flormaria1705@hotmail.com; https://orcid.org/0000-0003-4859-1279

2. Médico Especialista en Anestesiología; Investigador Independiente; Quito, Ecuador; tatyparafly79@gmail.com; (iD) https:// orcid.org/0000-0003-1821-9214

3. Médico General; Investigador Independiente; Quito, Ecuador; rosita1331lopez@gmail.com; (D) https://orcid.org/00000001-9750-8174

4. Médico General; Investigador Independiente; Quito, Ecuador; paopino3644@gmail.com; (ID) https://orcid.org/0000-00034995-3323

CORRESPONDENCIA

Flor María Pancha Ramos

dr.flormaria1705@hotmail.com

Quito, Ecuador 


\section{RESUMEN}

Introducción: La evaluación y predicción de una vía aérea difícil en el paciente pediátrico es de gran importancia para su correcto manejo, aplicación de algoritmos y disminución de complicaciones. Este estudio va dirigido a identificar los condicionantes de riesgo a través de la aplicación de la escala COPUR como herramienta fundamental para identificar la incidencia y problemas en la vía aérea pediátrica. Objetivos: Determinar la incidencia de vía aérea difícil e identificar los factores de riesgo en los pacientes pediátricos con cardiopatía de 1 día hasta 10 años, sometidos a cirugía bajo anestesia general a través de escala de COPUR. Metodología: Es un estudio analítico transversal realizado en 235 niños con cardiopatías congénitas de un Hospital Pediátrico en Quito- Ecuador sometidos a intervenciones quirúrgicas electivas bajo anestesia general en el período entre junio de 2015 y diciembre del 2016. Resultados: Se encontró una intubación normal y fácil en un 73.19\%, una mayor dificultad en la intubación y una necesaria maniobra de presión laríngea en el 24.26\% y una difícil intubación con requerimiento de dispositivos (fibroscopio o video laringoscopia) fue 2,25\%. Conclusión: La incidencia de vía aérea difícil fue del 2,25\% y los factores de riesgo como una hipoplasia mandibular, una apertura bucal menor $20 \mathrm{~mm}$, previa intubación difícil, una úvula parcialmente visible, y un rango de movimiento menor a 30 grados en conjunto nos permite una adecuada predicción de una vía aérea difícil, su anticipación permite el entrenamiento adecuado, el cumplimiento disciplinario del algoritmo y un mejor abordaje para una solución inmediata.

Palabras clave: Vía aérea difícil, intubación endotraqueal, niños, COPUR.

\section{ABSTRACT}

Introduction: The evaluation and prediction of a difficult airway in the pediatric patient is of great importance for its correct management, application of algorithms and reduction of complications. This study is aimed at identifying risk factors through the application of the COPUR scale as a fundamental tool to identify the incidence and problems in the pediatric airway. Objectives: To determine the incidence of difficult airway and identify risk factors in pediatric patients with heart disease from 1 day to 10 years old, who underwent surgery under general anesthesia through the COPUR scale. Methodology: This is a cross-sectional analytical study conducted in 235 children with congenital heart disease in a Pediatric Hospital in Quito-Ecuador undergoing elective surgery under general anesthesia in the period between June 2015 and December 2016. Results: Normal and easy intubation was found in 73.19\%, more difficult intubation and necessary laryngeal pressure maneuver in $24.26 \%$ and difficult intubation with requirement of devices (fiberscope or video laryngoscopy) was $2.25 \%$. Conclusion: The incidence of difficult airway was $2.25 \%$ and risk factors such as mandibular hypoplasia, a mouth opening smaller than $20 \mathrm{~mm}$, previous difficult intubation, a partially visible uvula, and a range of motion less than 30 degrees together allow an adequate prediction of a difficult airway, its anticipation allows adequate training, disciplined compliance with the algorithm and a better approach for an immediate solution.

Keywords: Difficult airway, endotracheal intubation, children, COPUR.

\section{RESUMO}

Introdução: A avaliação e previsão de uma via aérea difícil no paciente pediátrico é de grande importância para seu correto manejo, aplicação de algoritmos e redução de complicações. Este estudo tem como objetivo identificar fatores de risco através da aplicação da escala COPUR como ferramenta fundamental para identificar a incidência e os problemas na via aérea pediátrica. Objetivos: Determinar a incidência de vias aéreas difíceis e identificar fatores de risco em pacientes pediátricos com doenças cardíacas de 1 dia a 10 anos de idade, submetidos à cirurgia sob anestesia geral através da escala COPUR. Metodologia: Este é um estudo analítico transversal realizado em 235 crianças com cardiopatias congênitas em um Hospital Pediátrico em Quito-Equador submetido à cirurgia eletiva sob anestesia geral no período entre junho de 2015 e dezembro de 2016. Resultados: A intubação normal e fácil foi encontrada em 73,19\%, a intubação mais difícil e a necessária manobra de pressão laríngea em 24,26\% e a difícil intubação com necessidade de dispositivos (fibroscópio ou vídeo-laringoscopia) foi de 2,25\%. Conclusão: A incidência de vias aéreas difíceis foi de 2,25\% e fatores de risco como hipoplasia mandibular, uma abertura bucal menor que $20 \mathrm{~mm}$, intubação difícil anterior, uma úvula parcialmente visível e uma amplitude de movimento inferior a 30 graus juntos permitem uma previsão adequada de uma via aérea difícil, sua antecipação permite um treinamento adequado, cumprimento disciplinado do algoritmo e uma melhor abordagem para uma solução imediata.

Palavras-chave: Vias aéreas difíceis, intubação endotraqueal, crianças, COPUR. 


\section{Introducción}

El paciente pediátrico presenta diferencias anatómicas y fisiológicas marcadas en su vía aérea con respecto al adulto lo que permite en algunos casos dificultad en el abordaje. La vía aérea difícil se define como la presencia de factores clínicos que complican la ventilación con mascarilla facial o el proceso de intubación endotraqueal por una persona experimentada ${ }^{1-14}$. La prevalencia de vía aérea pediátrica se encuentra entre un $0,08 \%$ a $1,1 \%$ siendo algo mayor en niños menores de 1 año $(3,5 \%)^{2}$.

Las complicaciones relacionadas con el manejo de la vía aérea pediátrica pueden generar morbi-mortalidad entre estos problemas respiratorios pos-anestésicos en un 13\% 3,7 como causa directa se encuentra la intubación difícil, ventilación inadecuada.

La mayor parte de las complicaciones son evitables con una adecuada previsión del manejo y conocimiento de un posible problema de intubación endotraqueal es por eso la importancia de esta investigación es poder anticiparnos a una vía aérea difícil pediátrica a través de predictores de la escala de COPUR que nos permita un correcto abordaje, aplicación de los algoritmos de vía aérea difícil y posteriormente reducir las complicaciones pos-anestésicas.

\section{Objetivos}

- Determinar la incidencia de vía aérea difícil en los pacientes pediátricos con cardiopatía congénita de 1 día hasta 10 años de edad, sometidos de cirugía electiva bajo anestesia general a través de Cormack-Lehane y número de intentos de intubación endotraqueal.

- Evaluar los factores de riesgo de vía aérea difícil en los pacientes pediátricos cardiópatas de 1 día hasta 10 años de edad, intervenido a cirugía electiva con anestesia general a través de la escala de COPUR.
- Correlacionar la escala de COPUR con la presencia de vía aérea difícil (índice de Cormack-Lehane, intentos de intubación orotraqueal).

\section{Metodología}

\section{DISEÑO DE ESTUDIO}

Estudio analítico transversal

\section{UNIVERSO Y MUESTRA}

UNIVERSO: 8000 niños Hospital Pediátrico Quito-Ecuador intervenidos quirúrgicamente de manera electiva en el que se ha decidido el plan de anestesia general en el período de junio de 2015 a diciembre del 2016.

\section{TAMAÑO MUESTRAL}

Tamaño del Universo: 8000 niños

Heterogeneidad:50\% Margen de error:5\%

Intervalo de Confianza: 95\% Muestra: 198 niños:

- Proporción esperada de pérdidas: $15 \%$

- Muestra ajustada a pérdidas: 233 niños

Participantes del estudio: 235 niños con cardiopatías congénitas a través del Hospital Pediátrico Quito-Ecuador sometidos a intervenciones quirúrgicas electivas bajo anestesia general en el período entre junio de 2015 diciembre del 2016.

CRITERIOS DE INCLUSION Y EXCLUSIÓN 
Tabla 1. Criterios de Inclusión y exclusión del estudio

\begin{tabular}{|l|l|}
\hline CRITERIOS DE INCLUSION & CRITERIOS DE EXCLUSION \\
\hline Niños entre 1 día a 10 años. & $\begin{array}{l}\text { Niños mayores de 10 años } \\
\text { (adolescentes) }\end{array}$ \\
\hline $\begin{array}{l}\text { Pacientes pediátricos que no } \\
\text { presentan malformaciones } \\
\text { anatómicas en región facial, } \\
\text { columna cervical. }\end{array}$ & $\begin{array}{l}\text { Pacientes pediátricos que } \\
\text { presentan malformaciones } \\
\text { anatómicas en región facial, } \\
\text { columna cervical. }\end{array}$ \\
\hline $\begin{array}{l}\text { Pacientes sin evidencia de } \\
\text { síndromes genéticos específicos } \\
\text { como Pierre Robin, Síndrome de } \\
\text { Klippel Feil. Etc. }\end{array}$ & $\begin{array}{l}\text { Pacientes con evidencia de } \\
\text { síndromes genéticos específicos } \\
\text { como Pierre Robin, Síndrome de }\end{array}$ \\
\hline Estado Físico ASA I-II -III-IV & Klippel Feil etc. \\
\hline Cirugías Electiva & Cirugías de Emergencia \\
\hline
\end{tabular}

\section{TECNICAS -INSTRUMENTOS:}

\section{ESCALA DE COPUR}

Fue creada por el Dr. Geoffrey Lane establecido en el departamento de anestesiología de la Universidad del Colorado y aplicada por primera vez en el centro de ciencias de la Salud y Hospital de niños de Denver, Colorado. ${ }^{14}$ La Escala de COPUR que toma las variables fisiológicas y anatómicas a través del acrónimo descrito de la siguiente forma:

\section{Tabla 2. Escala COPUR}

\begin{tabular}{|l|l|}
\hline CHIN & $\begin{array}{l}\text { Mentón Se observa de forma lateral y observando un mentón } \\
\text { normal retroceso, hipoplasia moderada o extrema del mentón. }\end{array}$ \\
\hline OPENING & $\begin{array}{l}\text { Apertura Bucal espacio interdental de las piezas dentarias } \\
\text { situadas en la parte de adelante o espacio entre el maxilar } \\
\text { superior e inferior. }\end{array}$ \\
\hline PREVIOUS & $\begin{array}{l}\text { Previa intubación o historia apnea obstructiva del sueño } \\
\text { Úvula Visibilidad completa, parcial, insinuada visualización de } \\
\text { la úvula }\end{array}$ \\
\hline UVULA & $\begin{array}{l}\text { Observar la línea entre el ojo y la órbita, mirando el movimiento } \\
\text { hacia arriba o hacia abajo ya valoramos la angulación. }\end{array}$ \\
\hline RANGO & \\
\hline
\end{tabular}

Las modificaciones incluyen dientes prominentes frontales, macroglosia, obesidad, mucopolisacáridos pueden adicionarse 1 o 2 puntos más extras. Anexo 1.

Cada ítem tiene 4 opciones de respuesta con su respectiva puntuación de 1 a 4, la suma total se clasifica en 5 puntajes cada uno con la predicción de intubación y una recomendación de la técnica y dispositivo a utilizarse: 
Tabla 3. Escala COPUR

\begin{tabular}{|c|c|c|}
\hline PUNTAJE & PREDICTIVO & TECNICA Y DISPOSITIVO \\
\hline 5 a 7 & Normal y fácil intubación & Laringoscopia \\
\hline 8 a 10 & Ayuda de presión laríngea & Laringoscopia \\
\hline 11 a 12 & $\begin{array}{l}\text { Mayor dificultad puede ser una intubación } \\
\text { traumática }\end{array}$ & Utilización de Fibra óptica \\
\hline 13 a 14 & Difícil Intubación & $\begin{array}{l}\text { Utilización de Fibra óptica o } \\
\text { la utilización de otra técnica } \\
\text { avanzada. }\end{array}$ \\
\hline 15 a 16 & Peligrosa Intubación & $\begin{array}{lll}\text { Intubación con } & \text { paciente } \\
\text { despierto y y } & \text { potencial } \\
\text { traqueostomía. } & \end{array}$ \\
\hline
\end{tabular}

\section{CLASIFICACIÓN DE CORMACK-LEHANE}

Valora el grado de dificultad para la intubación endotraqueal al realizar la laringoscopia directa, según las estructuras anatómicas que se visualicen

- Grado I. Se observa el anillo glótico en su totalidad (intubación muy fácil).

- Grado II. Sólo se observa la comisura o mitad posterior del anillo glótico (cierto grado de dificultad)

- Grado III. Sólo se observa la epiglotis sin visualizar orificio glótico (intubación muy difícil pero posible)

- Grado IV. Imposibilidad para visualizar incluso la epiglotis (intubación sólo posible con técnicas especiales.

Se evaluaron 235 niños del Hospital Pediátrico Baca Ortiz sometidos a intervenciones quirúrgicas electivas bajo anestesia general. Se efectúo el chequeo pre-anestésico en el que se realizó la valoración de la vía aérea a través de la utilización del score COPUR, además se tomó en cuenta los datos demográficos y de filiación de cada uno de los pacientes.

Todos los datos tomados de la valoración pre-anestésica fueron ingresados al programa de microsoft excel. Las diferentes variables fueron expresadas en medidas de tendencia central con sus respectivos intervalos de confianza y posterior estadística inferencial.

INTENTOS DE INTUBACION

Número de intentos de intubación.

\section{RESULTADOS Y ANALISIS}

Participaron en el estudio 235 pacientes que representa el 100\% del tamaño muestral calculado, $53.62 \%$ eran niñas y $46.38 \%$ niños. Tabla 4

Tabla 4. Distribución por sexo del paciente

\begin{tabular}{|c|c|c|c|c|}
\hline SEXO & (n) & $\%$ & \multicolumn{2}{|c|}{ IC 95\% } \\
\hline Femenino & 126 & $53,62 \%$ & $47,02 \%$ & $60,12 \%$ \\
\hline Masculino & 109 & $46,38 \%$ & $39,88 \%$ & $52,98 \%$ \\
\hline Total & 235 & $100,00 \%$ & & \\
\hline
\end{tabular}


La edad de los participantes se encontraba en un rango de 1 día hasta los 10 años. La misma que fue clasificada de acuerdo a la edad pediátrica de la Organización Mundial de Salud O.M.S., se encontró un mayor porcentaje de pacientes entre el grupo de niños 56.17\% (IC95\% 49,39\%-63.43\%) y lactantes 36.17\% (IC95\% 36,17-30,02\%).

La edad promedio fue 53,02 +/- 50,1 meses (4años). El peso promedio fue 15,9 +/- 12,2 kilogramos. Tabla 5.

Tabla 5. Edad Pediátrica O.M.S.

\begin{tabular}{|c|c|c|c|c|}
\hline $\begin{array}{c}\text { EDAD } \\
\text { PEDIATRICA }\end{array}$ & (n) & $\%$ & \multicolumn{2}{|c|}{ IC 95\% } \\
\hline Neonato & 18 & $7,66 \%$ & $4,60 \%$ & $11,84 \%$ \\
\hline Lactante & 85 & $36,17 \%$ & $30,02 \%$ & $42,67 \%$ \\
\hline Niño & 132 & $56,17 \%$ & $49,39 \%$ & $63,43 \%$ \\
\hline Total & 235 & $100,00 \%$ & & \\
\hline
\end{tabular}

El estado físico fue catalogado de acuerdo a los criterios del ASA de los pacientes que predominó Tipo ASA III en un 70.21\% y ASA IV 14.89\% considerando que son pacientes con diagnóstico de cardiopatías congénitas complejas intervenidos quirúrgicamente con procedimientos correctivos y algunos casos procedimientos paliativos.

Tabla 6. Estado Físico ASA

\begin{tabular}{|c|c|c|c|c|}
\hline ASA & (n) & \% & \multicolumn{2}{|c|}{ IC } \\
\hline I & 6 & $2,55 \%$ & $0,94 \%$ & $5,47 \%$ \\
\hline II & 29 & $12,34 \%$ & $8,42 \%$ & $17,24 \%$ \\
\hline III & 165 & $70,21 \%$ & $63,92 \%$ & $75,98 \%$ \\
\hline IV & 35 & $14,89 \%$ & $10,60 \%$ & $20,10 \%$ \\
\hline Total & 235 & $100,00 \%$ & $100,00 \%$ & \\
\hline
\end{tabular}

\section{ESCALA DE COPUR}

En la tabla N7 se presentan los resultados de las evaluaciones de cada una de las variables de la escala COPUR observamos la distribución de los pacientes con sus puntuaciones.

Tabla 7. Parámetros de Escala COPUR

\begin{tabular}{|c|c|c|c|c|c|}
\hline & & (n) & $\%$ & & \\
\hline \multirow{3}{*}{ C MENTON } & 1 & 195 & $82,98 \%$ & $77,55 \%$ & $87,55 \%$ \\
\hline & 2 & 38 & $16,17 \%$ & $11,70 \%$ & $21,51 \%$ \\
\hline & 3 & 2 & $0,85 \%$ & $0,10 \%$ & $3,04 \%$ \\
\hline \multirow{3}{*}{$\begin{array}{l}\text { O APERTURA } \\
\text { BUCAL }\end{array}$} & 1 & 151 & $64,26 \%$ & $57,77 \%$ & $70,38 \%$ \\
\hline & 2 & 66 & $28,09 \%$ & $22,44 \%$ & $34,30 \%$ \\
\hline & 3 & 15 & $6,38 \%$ & $3,62 \%$ & $10,31 \%$ \\
\hline
\end{tabular}




\begin{tabular}{|c|c|c|c|c|c|}
\hline & 4 & 3 & $1,28 \%$ & $0,26 \%$ & $3,69 \%$ \\
\hline \multirow{3}{*}{$\begin{array}{l}\text { P PREVIAS } \\
\text { INTUBACIO } \\
\mathbf{N}\end{array}$} & 1 & 80 & $34,04 \%$ & $28,01 \%$ & $40,49 \%$ \\
\hline & 2 & 148 & $62,98 \%$ & $56,46 \%$ & $69,17 \%$ \\
\hline & 3 & 7 & $2,98 \%$ & $1,21 \%$ & $6,04 \%$ \\
\hline \multirow{3}{*}{ U UVULA } & 1 & 176 & $75,22 \%$ & $68,72 \%$ & $80,22 \%$ \\
\hline & 2 & 54 & $23,08 \%$ & $17,84 \%$ & $29,01 \%$ \\
\hline & 3 & 4 & $1,71 \%$ & $0,47 \%$ & $4,32 \%$ \\
\hline \multirow{3}{*}{$\begin{array}{l}\text { R RANGO } \\
\text { CERVICAL }\end{array}$} & 1 & 164 & $70,09 \%$ & $63,78 \%$ & $75,88 \%$ \\
\hline & 2 & 44 & $18,80 \%$ & $14,01 \%$ & $24,41 \%$ \\
\hline & 3 & 26 & $11,11 \%$ & $7,39 \%$ & $15,86 \%$ \\
\hline
\end{tabular}

La evaluación de cada uno de las cinco variables de la Escala de COPUR tiene una puntuación total que nos permite agrupar en 5 estados predictivos. Tabla 8.

Tabla 8. Puntuación de escala COPUR

\begin{tabular}{|c|c|c|c|c|}
\hline $\begin{array}{c}\text { PUNTUACION } \\
\text { TOTAL }\end{array}$ & "n" & \% & \multicolumn{2}{|c|}{ IC } \\
\hline $\mathbf{5}$ & 25 & $10,64 \%$ & $7,00 \%$ & $15,30 \%$ \\
\hline $\mathbf{6}$ & 88 & $37,45 \%$ & $31,24 \%$ & $43,97 \%$ \\
\hline $\mathbf{7}$ & 57 & $24,26 \%$ & $18,92 \%$ & $30,25 \%$ \\
\hline $\mathbf{8}$ & 28 & $11,91 \%$ & $8,07 \%$ & $16,76 \%$ \\
\hline $\mathbf{9}$ & 21 & $8,94 \%$ & $5,62 \%$ & $13,34 \%$ \\
\hline $\mathbf{1 0}$ & 10 & $4,26 \%$ & $2,06 \%$ & $7,69 \%$ \\
\hline $\mathbf{1 1}$ & 1 & $0,43 \%$ & $0,01 \%$ & $2,35 \%$ \\
\hline $\mathbf{1 2}$ & 1 & $0,43 \%$ & $0,01 \%$ & $2,35 \%$ \\
\hline $\mathbf{1 3}$ & 4 & $1,70 \%$ & $0,47 \%$ & $4,30 \%$ \\
\hline Total & 235 & $100,00 \%$ & & \\
\hline & & & & \\
\hline
\end{tabular}

En el presente estudio se observó una predicción de intubación normal y fácil sin ningún contratiempo un $72.34 \%$ de pacientes, una mayor dificultad en el momento de la intubación y una necesaria maniobra de presión laríngea en el 25,10\% y aquellos en el que se presenta una difícil intubación y se requirió del uso de dispositivos para vía aérea difícil como fibroscopio o video laringoscopia fue $2,55 \%$. Tabla 9

Tabla 9. Interpretación de escala COPUR

\begin{tabular}{|c|c|c|c|c|}
\hline INTERPRETACION & "n" & $\%$ & \multicolumn{2}{|c|}{ IC 95\% } \\
\hline $\begin{array}{c}\text { Difícil Intubación/Fibra } \\
\text { óptica }\end{array}$ & 6 & $2,55 \%$ & $0,94 \%$ & $5,47 \%$ \\
\hline $\begin{array}{c}\text { Mayor dificultad } \\
\text { /presión laríngea }\end{array}$ & 59 & $25,10 \%$ & $18,92 \%$ & $30,25 \%$ \\
\hline Normal Intubación & 170 & $72,34 \%$ & $67,05 \%$ & $78,74 \%$ \\
\hline Total & 235 & $100,00 \%$ & & \\
\hline
\end{tabular}


La escala nos ayuda a predecir una posible presencia de vía aérea difícil que la corroboramos en el momento de la realización de la laringoscopia directa a través de la Escala de Cormack Lehane en el que observamos que la mayoría de pacientes nos mostraron 57.87\% ,34,89\%,6.81\% y $0.43 \%$ respectivamente al Grado I, II, III, IV.

Tabla 10. Cormack - Lehane

\begin{tabular}{|c|c|c|c|c|}
\hline $\begin{array}{c}\text { CORMAC } \\
\text { K- } \\
\text { LEHANE }\end{array}$ & "n" & \% & \multicolumn{2}{|c|}{$\begin{array}{c}\text { IC } \\
\mathbf{9 5} \%\end{array}$} \\
\hline $\mathbf{1}$ & 136 & $57,87 \%$ & $51,28 \%$ & $64,26 \%$ \\
\hline $\mathbf{2}$ & 82 & $34,89 \%$ & $28,81 \%$ & $41,36 \%$ \\
\hline $\mathbf{3}$ & 16 & $6,81 \%$ & $3,94 \%$ & $10,82 \%$ \\
\hline $\mathbf{4}$ & 1 & $0,43 \%$ & $0,01 \%$ & $2,35 \%$ \\
\hline Total & 235 & $100,00 \%$ & & \\
\hline
\end{tabular}

Los grados III y IV de Cormack-Lehane se encontraron finalmente en edades pediátricas de niños y lactantes como se observa a continuación:

Tabla 11. Correlación de Cormack - Lehane con edades pediátricas

\begin{tabular}{|c|c|c|c|c|c|c|c|c|c|}
\hline \multirow{3}{*}{$\begin{array}{c}\text { EDAD } \\
\text { PEDIATRICA }\end{array}$} & \multicolumn{8}{|c|}{ CORMACK - LEHANE } & \multirow{3}{*}{$p$} \\
\hline & \multicolumn{2}{|c|}{1} & \multicolumn{2}{|c|}{2} & \multicolumn{2}{|c|}{3} & \multicolumn{2}{|c|}{4} & \\
\hline & "n" & $\%$ & "n" & $\%$ & "n" & $\%$ & "n" & $\%$ & \\
\hline Neonato & 3 & $16.7 \%$ & 15 & $83.33 \%$ & 0 & $0.0 \%$ & 0 & $0.0 \%$ & \multirow{3}{*}{0,0001} \\
\hline Lactante & 38 & $44.7 \%$ & 36 & $42.3 \%$ & 9 & $10.6 \%$ & 2 & $2.4 \%$ & \\
\hline Niño & 95 & $72 \%$ & 31 & $23,5 \%$ & 5 & $2,8 \%$ & 1 & $0.8 \%$ & \\
\hline
\end{tabular}

Se evidenció la presencia 17 pacientes que presentaron grado III-IV de la escala de Cormack- Lehane.

En los 17 pacientes se requirió más de 2 intentos de intubación:

- En 12 pacientes de ellos se requiere 2 intentos de intubación endotraqueal con laringoscopia directa y maniobra BURP.

- En 5 pacientes se efectúo 3 intentos de intubación endotraqueal en su tercer intento usamos una técnica alternativa a la laringoscopia directa usando video-laringoscopio en el que logramos una intubación endotraqueal exitosa.

Tabla 12. Intentos de intubación

\begin{tabular}{|c|c|c|c|c|}
\hline $\begin{array}{l}\text { INTENTOS } \\
\text { DE } \\
\text { INTUBACION }\end{array}$ & "n" & $\%$ & \multicolumn{2}{|c|}{$\begin{array}{c}\text { IC } \\
\mathbf{9 5 \%}\end{array}$} \\
\hline $\mathbf{1}$ & 218 & $92,77 \%$ & $89,18 \%$ & $96,06 \%$ \\
\hline$>\mathbf{2}$ & 17 & $7,23 \%$ & $3,94 \%$ & $10,82 \%$ \\
\hline Total & 235 & $100,00 \%$ & & \\
\hline
\end{tabular}




\section{CORRELACION DE ESCALA COPUR}

Para determinar el impacto que tienen la identificación de los factores de riesgo evaluados COPUR con el grado de Cormack-Lehane encontramos los siguientes hallazgos:

- Los pacientes que presentaron una puntuación de 11 a 12 en la escala COPUR y se predijo una difícil intubación con necesaria ayuda de fibra óptica fue de 6 pacientes de los cuales 5 niños presentaron en realidad en el momento de la laringoscopia una difícil intubación con un Cormack-Lehane grado III-IV.

- Una puntuación de la escala de COPUR de 8 a 10 en el que su predicción es una mayor dificultad en la intubación endotraqueal en el que se requiere de la maniobra de BURP se predijo en 59 pacientes pero el hallazgo fue mayor y se encontraron 64 pacientes con grado de Cormack-Lehane grado I, II, III.

- Se estimó una intubación fácil y sin problemas en 172 pacientes, pero solo en 136 pacientes se evidenció una intubación normal.

\begin{tabular}{|c|c|c|c|c|c|c|c|}
\hline \multirow{3}{*}{$\begin{array}{l}\text { CORMACK - } \\
\text { LEHANE }\end{array}$} & \multicolumn{6}{|c|}{ INTERPRETACION } & \multirow{3}{*}{$P$} \\
\hline & \multicolumn{2}{|c|}{$\begin{array}{c}\text { Difícil } \\
\text { intubación/Fibra } \\
\text { óptica }\end{array}$} & \multicolumn{2}{|c|}{$\begin{array}{l}\text { Mayor dificultad } \\
\text { /presión laríngea }\end{array}$} & \multicolumn{2}{|c|}{$\begin{array}{c}\text { Normal } \\
\text { intubación }\end{array}$} & \\
\hline & "n" & $\%$ & "n" & $\%$ & "n" & $\%$ & \\
\hline 1 & 0 & $0.0 \%$ & 28 & $20.6 \%$ & 108 & $79.4 \%$ & \multirow{4}{*}{0,0001} \\
\hline 2 & 0 & $0.0 \%$ & 25 & $30,48 \%$ & 57 & $69.5 \%$ & \\
\hline 3 & 5 & $31,25 \%$ & 11 & $68,75 \%$ & 0 & $0.0 \%$ & \\
\hline 4 & 1 & $100.0 \%$ & 0 & $0.0 \%$ & 0 & $0.0 \%$ & \\
\hline
\end{tabular}

\section{Discusion}

La investigación se realizó en los 235 pacientes sin aparentes síndromes genéticos o alteraciones craneofaciales que puedan predecir una vía aérea difícil anticipada, pero llegamos a encontrar que los pacientes que no tienen predictores de vía aérea difícil anticipada o sospechosa pueden tener una vía aérea difícil inesperada9

La presencia de pacientes pediátricos con dificultad en la ventilación con mascarilla facial fue nula en nuestro estudio como se reportan en algunas revisiones. 9 No hay evidencia de dificultad en la ventilación con mascarilla facial incluso en aquellos pacientes que presentaron predictivos (COPUR) de vía aérea difícil por lo que no se presentaron problemas de oxigenación e hipoxia incluso en pacientes con diagnósticos de cardiopatías cianóticas.

La edad pediátrica que se incluyó fue 7,66\% neonatos, $36,17 \%$ lactantes, niños 56,17\% se abarcó un amplio grupo de edades a diferencia de la mayoría de estudios en el que se incluyen pacientes de edades superiores al primer año por una mejor comprensión y colaboración en las pruebas a partir de esa edad.7 En Alemania, un estudio retrospectivo de 11.219 anestesias generales pediátricas mostraron una mayor dificultad en la laringoscopia en menores de 1 año12 a diferencia de nuestro estudio la incidencia de vía aérea difícil se observó en edades comprendidas entre de 1.6 a 2 años.

La aparición de complicaciones se asoció con más de dos intentos de intubación traqueal en menores con un peso comprendi- 
do entre 10 a 15 kilogramos en promedio en un estudio en los E.E.U.U. esto asociación se presentó en niños de un peso de menos de $10 \mathrm{~kg}^{17}$.

Al igual que el estado físico ASA predomina el ASA III (70.21\%) y IV (14.84\%) por la inclusión de pacientes con cardiopatías congénitas complejas. Un estudio retrospectivo en un centro universitario en el que se observó una vía aérea difícil en pacientes pediátricos presentó el mismo estado físico ASA III y IV en intervenciones quirúrgicas cardíacas, se observó en este caso escala de Mallampati grado III y IV ${ }^{12,15}$.

El grado de Cormack-Lehane se encontró una mayor incidencia el grado I y II. Las complicaciones en la intubación endotraqueal se definió en un grado III (6\%) y grado IV $(0.4 \%)$ a diferencia de un estudio realizado en 100 niños en New Delhi en el que su incidencia fue menos del $3 \% 7$ en un segundo estudio retrospectivo en Alemania de 177 niños sometidos a cirugía cardiaca fue de $3.5 \%$ de manera global pero en niños de 1 año esta incidencia de grado III y IV se encuentra en un rango cercano de grado III $(5.6 \%)$ y grado IV $(1.7 \%)$. Estos hallazgos no se presentaron en pacientes con síndrome de Down en el no tuvieron ningún problema durante la laringoscopia, pero si en síndrome de George o síndrome CHARGE ${ }^{13}$.

Los predictores que han mostrado buena sensibilidad y especificidad, como la protrusión mandibular, la clasificación de Mallampati y extensión de la articulación atlanto-occipital son muy difíciles de evaluar en los niños menores de 3 años ${ }^{9}$. en el presente estudio está enfocado analizar estos factores de la escala de COPUR que nos permita valorar incluso en niños menores de 1 año en estos esta incluido la estructura mandibular, la evidencia de la úvula y la angulación cervical a partir de la línea trago auricular-orbita ${ }^{7}$.
La distancia intermaxilar o el espacio entre los incisivos superiores e inferiores, la distancia tiromentoniana, la apertura oral la cual es adecuada si caben 2 dedos del mismo y las alteraciones en mandíbula, como retrognatia, micrognatia y prognatismo son más fáciles de valorar en los niños eso se observado en revisiones de estudios del 2001-2015 en países como República Checa ${ }^{9,10}$, en esta investigación hemos tomado en cuenta la distancia intermaxilar o espacio interdental $>40 \mathrm{~mm}$ en un $28 \%$ y entre $20 \mathrm{~mm}$ a $40 \mathrm{~mm}$ en un $6 \%$ la presencia de una lengua grande se observado en los lactantes y en niños preescolares y escolares la presencia de adenoides y amígdalas grandes contribuyen a una reducción o pérdida de este espacio en nuestro estudio.

La incidencia en nuestro estudio de una franca vía aérea difícil con grado III-IV de Cormack Lehane y más de 2 intentos de intubación endotraqueal fallidos en el que se logró una intubación endotraqueal exitosa a través de video laringoscopia es $2 \%(n=5)$ la incidencia estimada en un estudio en Colombia en menores de 1 año es $0.6 \%$ y van disminuyendo conforme aumenta la edad.9

La incidencia de vía aérea difícil en este estudio fue del $2 \%$ en comparación a un ensayo retrospectivo en 11.219 anestesias pediátricas en Alemania en el que el riesgo estimado fue de $1.35 \%, 12,14$ Partani et al ${ }^{16}$ en la India en 100 niños el riesgo 4\% y en 13 hospitales pediátricos en los E.E.U.U. en el periodo 2012 a 2015 se reporta un $2 \%{ }^{17}$.

\section{Conclusión}

La incidencia de vía aérea difícil fue del 2\% y los factores de riesgo de la escala de COPUR como una marcada hipoplasia mandibular, una apertura bucal menor $20 \mathrm{~mm}$, previa intubación con dificultad, una úvula parcialmente visible y paladar blando, y un rango de movimiento menor a 30 grados en conjunto nos permite una adecuada predicción de una vía aérea difícil en el paciente 
pediátrico, su anticipación permite el entrenamiento adecuado, el cumplimiento disciplinario del algoritmo y un mejor abordaje para una solución inmediata y exitosa.

\section{Bibliografía}

1. Alarcón J,Cano M. Asociación de los grados de movilidad de la articulación atlanto-occipital con la clasificación de Cormack-Lehane como predictores de una vía aérea pediátrica difícil intubación difícil mediante escalas de valoración de la vía aérea. Rev. Mex. Anestesiol.(Internet).2016;39(1):15-19. Disponible en:

2. Andreu E, Schmucker E. Algoritmo de la vía aérea difícil en pediatría. Rev Esp Anestesiol Reanim (Internet).2011; 58:304-311. Disponible en:

3. Vieira A, Andrade L, Telles M. Intubación Difícil en Niños: Aplicabilidad del Índice de Mallampati. Rev Bras Anestesiol .(Internet). 2011; 61: 2: 84-87. Disponible en:

4. Ramírez J, Torrico G. Índices predictores de vía aérea en pacientes obesos. Rev. Mex. Anestesiol (internet). 2013; 36(3):193-201. Disponible en:

5. Langeron O, Masso E, Prediction of difficult mask ventilation. Anesthesiology.(Internet). 2000;92(5):1229-1236. Disponible en:

6. Tay CL, Tan GM. Critical incidents in paediatric anaesthesia: an audit of 10.000 anaesthetics in Singapore. Paediatric Anaesthesia (Internet) 2001; 11: 711-718. Disponible en:

7. Anur A, Kavita R,. Evaluation of "Difficult Airway Predictors in Pediatric Population" As a Clinical Inv.J Anes Clinc Res (Internet). 2012; 3:1.

8. Belanger J, Kossick M.Methods of Identifying and managing the difficult airway in the pediatric population. AANA Journal(Internet).2015;83:35-41. Disponible en:
9. Echeverry PC, Engelhardt T. Algoritmo para el manejo de la vía aérea difícil en pediatría. Rev Col Anestesiol. (Internet) 2014; 42:325-334. Disponible en:

10. Fritscherovaa S ,Adamusa M. Can difficult intubation be easily and rapidly predicted?.Biomed Pap Med Fac Univ Palacky Olomouc Czech Repub. 2011 Jun; 155(2): 165-172

11. Ríos A, Gómez L,Aguirre O, Ospina Federico. La vía aérea pediátrica: algunos conceptos para tener en cuenta en el manejo anestésico. rev colomb anestesiol. 2012;40(3):199-202

12. Heinrich S1, Birkholz T, Ihmsen H, Irouschek $A$. Incidence and predictors of difficult laryngoscopy in 11,219 pediatric anesthesia procedures. Paediatr Anaesth. 2012 Aug;22(8):729-36.

13. Heinrich S1, Birkholz T, Inmsen H, Irouschek A, Ackermann A. Incidence and predictors of poor laryngoscopic view in children undergoing pediatric cardiac surgery. J Cardiothorac Vasc Anesth. 2013 Jun;27(3):516-21.

14. Raj D, Luginbuehl I. Managing the difficult airway in the syndromic child. Critical Care \& Pain 2015 ;15 (1) :7-13.

15. Harless J. Ramaiah R.Pediatric airway management. International Journal Critical IIIn Inj Sci. 2014 Jan-Mar; 4(1): 65-70.

16. Partani S , Sharma R. Study of various measurements of intubation in infants and their correlation with prediction of difficult intubation. J. Evid. Based Med. Healthc.,2016; Vol. 3(13):406-410.

17. Edem J, Nishisaki A,Narasimba J,Hunyady A,Greenberg R. Airway management complications in children with difficult tracheal intubation from the Pediatric Difficult Intubation (PeDI) registry: a prospective cohort analysis. The Lancet. January 2016; Volume 4,(1): p37-48.

\section{CITAR ESTE ARTICULO:}

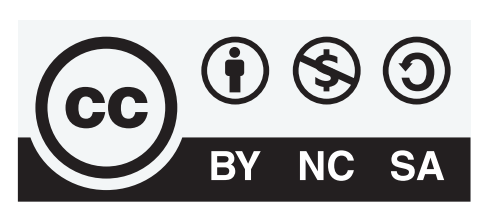

Pancha Ramos, F. M., Cevallos Pacheco, I. T., López Samaniego, R. D., \& Pino Vaca, D. P. (2021). Evaluación de predictores de vía aérea difícil en el paciente pediátrico. RECIMUNDO, 5(1), 153-163. https://doi.org/10.26820/recimundo/5.(1).enero.2021.153-163 